\title{
Research on cold bootstrapping method of Sparse User Comment in Online Trading Platform
}

\author{
Yuhua $\mathrm{Li}^{1, \mathrm{a}}$, Songlin $\mathrm{Liu}^{1, \mathrm{~b}}$ and Yang Pan ${ }^{2, \mathrm{c}}$ \\ ${ }^{1}$ The School of Statistics and Mathematics, Zhongnan University of Economics and Law, Wuhan \\ 430073, China \\ ${ }^{2}$ School of Management, Huazhong University of Science and Technology, Wuhan 430074, China \\ a4850237@qq.cm, broger0007@sina.com, 'panyang_25@hust.edu.cn
}

Keywords: E-commerce, User Evaluation, Product Credibility, Cold Boot.

\begin{abstract}
The trust value of the product is often through historical evaluation of calculated in online trading platform. However, some commodities have no or only a few of user evaluation, the traditional method of calculation will be very inaccurate and unfair trust value, seriously affecting the search results and users. The issue boils down to trust of the user evaluation sparse cold start problem by analyzing product providers, historical evaluation of the credibility of the user, as well as the characteristics of the product itself, the product structure features comprehensive network of trust, trust products to form an effective value estimation method. Experiments show that the method in the evaluation of a few rare cases remained good accuracy, and is not sensitive to malicious evaluation. This method can help users get real new products from trusted online trading platform.
\end{abstract}

\section{Introduction}

With the wide application of the Internet, online services and e-commerce to be more widely used. Because of the anonymity and uncertainty of the Internet, online trading service provider (seller) and goods and services can't be a user (buyer) intuitive, comprehensive understanding, both parties lack trust basis and conditions, making online transaction fraud, quality, security and other issues as a constraint and damage important issue of Internet development ${ }^{[1]}$. Currently online trading platform is mainly used in trust mechanism (reputation mechanism) to identify and describe the service providers and the reliability of goods and services, through the analysis of historical transaction records and evaluation records to predict the extent of commodity trustworthy for the new Buyers offer unbiased advice and reference, thus reducing the risk of online transactions ${ }^{[2]}$. And Trust Mechanism in Sort specifications, which directly affect the ranking of commodities and exposure, has very important significance.

Fair, accurate trust mechanism is an important factor in promoting the development of online trading platform, through effective assessment and recommendation, to improve user acceptance and satisfaction in the transaction process, thereby increasing the actual volume of transactions. At present research on trust mechanism is primarily about trust system architecture, user evaluation of the program, calculated the value of the trust, the main consideration trust evaluation algorithm complexity, and accuracy and impact assessment methods evaluated false ${ }^{[3]}$. For any kind of assessment methods, as long as there is enough real user reviews, can more effectively and accurately assess the value of commodities for the trust. However, many new products listed or non-hot commodity that only a small number of evaluation or no evaluation, or there is a lot of malicious evaluation and false, in which case the trust evaluation methods will have a huge error ${ }^{[4]}$. This user evaluation in only a few or no user evaluation of the situation on the commodity called trust trust evaluate cold start. The main problems and existing solutions that bring the main as follows:

(1) Incorrect trust cold start initial values affect the sales of products in the online trading platform. Search results are generally higher priority will trust the value of the top surface, the top surface of the product is more likely to be users to view and choose. If you trust the evaluation was not evaluated as a commodity even lower initial fixed value is 0 , it will cause a user of these products are difficult to 
see, even more difficult to generate new evaluation, resulting in Matthew. If these new products are actually very good, but no user is found, it is not only the loss of sales of businesses, but also the loss of user satisfaction with product selection opportunity in the long run for the normal development of the online trading platform is extremely negative, not promote the sound development of the platform.

(2) Will set up a trust cold start value to a larger value or maximum value will affect the fairness of the platform. This setting method has many drawbacks, because the trust value the more difficult it reaches a maximum evaluation of commodities, so this mechanism into the e-commerce system to unfavorable commodity; In addition, since the start value of high trust, shops in commodities After reducing the trust value re-register into the system which in turn has a high trust value.

(3) Separate analysis of the product itself and the trust value evaluation has limitations. In the absence of the evaluation, only a small amount calculated using the ratio of the maximum value of the trust property and similar affirmed actual property evaluation, this approach has its rationality, but subjectively believed the attribute values satisfy the linear relationship over the entire range worth exploring. After use. First of user evaluation means clustering classification, when calculating the trust value to the class members are mostly higher weights, but this method only relatively long time to be useful in the evaluation, but it is difficult to play a role only rarely evaluated. This approach such a problem is because the root cause assessment is limited to the evaluation of the product itself, as untrue, a higher proportion of malicious evaluation, it is difficult to rely on them to get an accurate assessment of local information.

In order to improve the accuracy of assessments trust value of goods, particularly in the absence of the evaluation and the evaluation of the assessment of the accuracy of small value their trust, the paper web from the user evaluation undertaken to assess the credibility of the user evaluation, under certain conditions, only a small amount even A user evaluation can accurately assess the value of goods trusted by the user evaluation, and the other by a combination of product evaluation stated property and commodities with other goods store, has been evaluated to assess the credibility is not high or no evaluation of the product trust value.

\section{Basic Theory}

In the existing trust evaluation model, based on the evaluation after the user purchases of goods made to evaluate the trust value of goods, various methods are different, but they have one thing in common is asked to evaluate more than the number, if less Number of Ratings and relatively large errors have higher proportion of malicious evaluation, these methods are evaluated within the scope of the assessment of the product itself, are vulnerable to their own false information, and malicious users evaluate the effect of the seller, but in fact, under certain conditions, even only one user evaluation, if the user reviews to know with sufficient certainty, can also be obtained accurately trust value of goods based on this evaluation.

For user $U$, is known he bought in TWS and the commodity composition of the ensemble as $P=\left\{p_{1}, p_{2}, \cdots, p_{n}\right\}, p_{i}$ corresponding to the quotient evaluation information collection is $C_{p i}=\left\{c_{p 1}, c_{p 2}, \ldots, c_{p m}\right\}$ these relationships constitute goods and Exposition evaluate social networks. According to the evaluation of the network, if the user's transactions are more on the user is able to effectively assess the credibility of comments.

\section{Cold Start for Trusted Computing}

When users search for goods e-commerce platform, the trust value of goods is an important parameter affecting commodity order. Trust value by the evaluation of the product, impact assessment of the credibility of the user, where the credibility of the product provider, the type of goods and their own description of the case and other information.

Evaluation reflects the trust of particular products can be expressed as: 


$$
T_{p}=\left(\sum_{k=1}^{n} T(k) \omega_{k}\right) / \sum_{k=1}^{n} \omega_{k}
$$

Where $\mathrm{n}$ represents the platform review content attribute dimension, $\omega_{k}$ represents the $\mathrm{k}$-th attribute weights, $T(k)$ indicates that the product value on the trust the k-th attribute. So the formula (1) weighted average trust value different attributes dimension. $T(k)$ can be expressed as follows:

$$
T(k)=\left(\sum_{j=1}^{m} r_{k}(j) \xi_{j}\right) / \sum_{j=1}^{m} \xi_{j}
$$

Where $\mathrm{m}$ is the number of the evaluation of the product, $r_{k}(j)$ represents the $\mathrm{j}$-th commodity standardized assessment scores, ranging from $[0,1], \xi_{j}$ represents the evaluation of credibility.

\section{Experimental Analysis}

Start using the trust is to make new products in line with user needs to get a chance in a large number of existing users to choose a number of evaluation records of commodity groups. The method used in this paper is sorted by attributes, attribute values of several relatively close linear relationship is more easily met. Through the acquisition expedia (expedia.com) online services and reviews the data, randomly selected samples for analysis, the data set for four categories comments: Room cleanliness, Service \& staff, Room comfort, Hotel condition for each evaluation item range is $\{1,2,3,4,5\}$.

Table 1 The number of product with different comment number by accurate evaluation

\begin{tabular}{ccc}
\hline Comment Number & Product Number & Percent \\
\hline$<10$ & 464 & $16.96 \%$ \\
{$[10,20)$} & 241 & $8.81 \%$ \\
{$[20,30)$} & 357 & $13.05 \%$ \\
{$[30,40)$} & 456 & $16.67 \%$ \\
{$[40,50)$} & 412 & $15.06 \%$ \\
{$[50,60)$} & 349 & $12.76 \%$ \\
$>=60$ & 457 & $16.70 \%$ \\
\hline
\end{tabular}

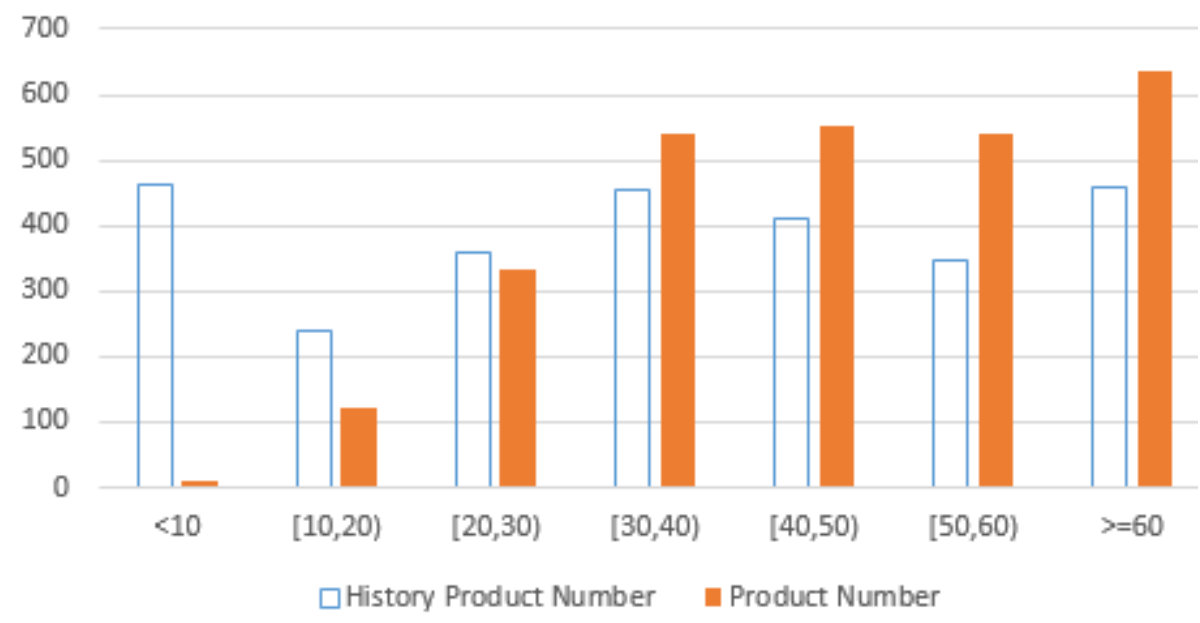

Fig. 1 The original data and new data number of products within the range of different comments

To test the predictive power of trust designed boot method, this article from Expedia randomly selected 2736 products over through user evaluation network can accurately assess the evaluation number, number of items and the number corresponding to each evaluation value of the product you trust the percentage of the total number of items as shown in Table 1. 
A confidence value calculated using the methods described herein by the data set and calculate trust value after a period of time at a few of these data to evaluate meet the conditions of the conventional method, and as a true value to be analyzed, in order to achieve comparing the value of the trust, the paper reviews several different trust value is calculated, the number of comments that the data set is shown in the Fig. 1 after 1 month:

Based on this evaluation to analyze the trust value, the original value and the real trust trust value calculation, and the error solved each product, a mean value of the original data set of the original trust, the average real value and the average error of the trust shown in Figure 2 :

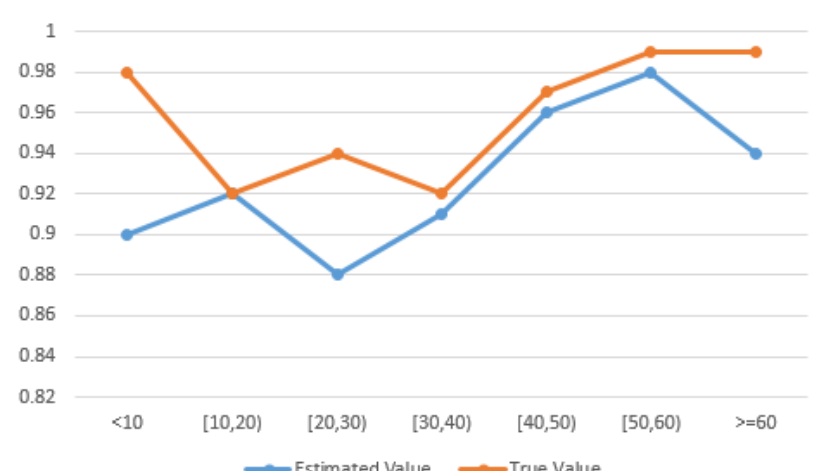

$\multimap$ Estimated Value $\multimap$ True Value

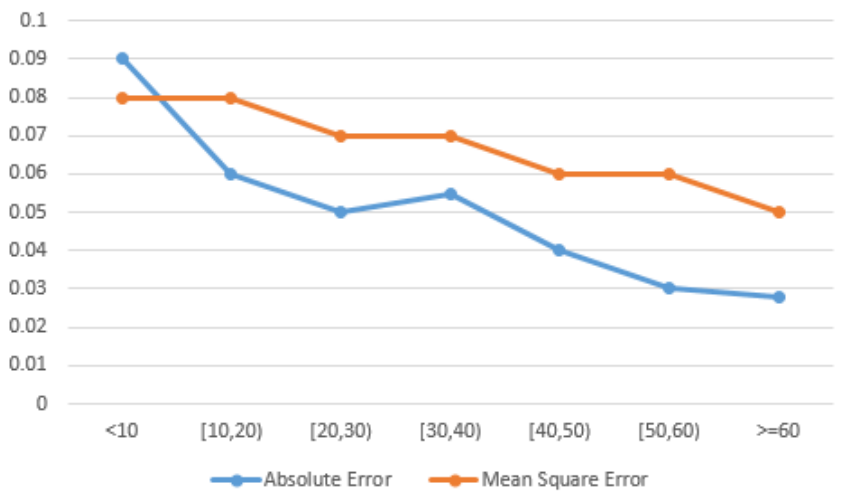

(a) Comparison between Estimated Value and True Value (b) Absolute Error and MeanSquare Error Fig.2 Comparison of Precision

Experimental results show that the number of comments a few cases, the proposed method can effectively still trust value product estimates. Although in the case of a large number of comments, the deviation value of the trust will become increasingly smaller, but in the case of insufficient number of comments, the estimated value of the trust to meet the requirements of error, with good accuracy.

\section{Summary}

Start a matter of trust evaluate all must face, and trust in e-commerce due to start directly related to money is very important. This paper studies the network through the evaluation of assessing and evaluating the credibility of the evaluation so that only few can accurately assess the value of the commodity trust, while trust commodity affirm properties with similar product attributes contrast and other goods in the shops means of assessing transfer no assessment or evaluation of the credibility of the case is not high by calculation, testing and analysis, we show that the method can effectively solve the initial product was added to the problem difficult to accurately assess the value of trust.

\section{References}

[1]. Winston T. Lin, Yueh H. Chen, Benjamin B. M. Shao, Assessing the business values of information technology and e-commerce independently and jointly. EUROPEAN JOURNAL OF OPERATIONAL RESEARCH. Vol 245(2015) No.3,p.815-827

[2]. Dongjuan Wang, Research on Trust Risk of P2P E-commerce, World Automation Congress. Puerto Vallarta, JUN 24-28, 2012

[3]. Xu Yongzhi, Sun Lu, Research on Consumer Confidence under the Online Shopping, International Conference on Innovative Computing and Communication/Asia-Pacific Conference on Information Technology and Ocean Engineering, Macao, JAN 30-31, 2010, 366-369.

[4]. Zainab M. Aljazzaf, Miriam A. M. Capretz, Trust bootstrapping services and service providers,2011 9th Annual International Conference on Privacy, Security and Trust, Canada, July 19, 2011, p 7-15 